\title{
HUSSERL EN EL PENSAMIENTO \\ DE HANNAH ARENDT
}

Agustín Serrano de Haro

CSIC

1. La poderosísima presencia de la persona y la obra de Heidegger sobre la persona y el pensamiento de Hannah Arendt es un hecho tan sabido que apenas hay monografia dedicada a la pensadora judía que no se ocupe con cierto detalle de sopesar el alcance de esta influencia. Ciertamente que a este respecto se produce un verdadero conflicto de interpretaciones, en el que combaten quienes ven en Arendt a "una hija de Heidegger" contra quienes, en el otro extremo, defienden que "la hija de Tracia" se separa radicalmente "del pensador profesional" que había sido su maestro en Marburgo. ${ }^{1}$ Para los primeros Arendt comparte en todo lo esencial la nostalgia incurable de lo griego originario y hereda el movimiento inequívoco de rechazo de la Modernidad, mientras que para los segundos la concepción arendtiana de la acción política participativa como ámbito de la pluralidad irreductible de los hombres y de realización precaria de la libertad la sitúa muy lejos del pensar del Ser. Este debate, que atrae masivamente la atención de estudiosos e intérpretes, es fundamental. Pero su importancia no debiera condenar a la irrelevancia, ni menos a la inexistencia, todo posible examen de la relación entre la obra de Arendt y el pensamiento de Husserl, siquiera fuera por esa trivial transitividad de que la obra de Husserl está a su vez en el origen polémico del pensar de Heidegger.

Sobre esta otra cuestión, que sólo en apariencia responde a un interés erudito, apenas si ha avanzado algo el trabajo de la crítica. Resulta significativo que ni siquiera la circunstancia biográfica de la estancia de Arendt en

Vid. como referentes emblemáticos: Jacques Taminaux, La fille de Trace et le penseur professionnel. Arendt et Heidegger (Paris, Payot, 1992) y. Richard Wolin, Heidegger's Children (Prensas de la Universidad de Princeton, 2001 -hay trad. cast: Los hijos de Heidegger, Madrid, Cátedra, 2003-). 
Friburgo como estudiante de Husserl se halle claramente comprobada y precisada. La magna Husserl-Chronik de Karl Schuhmann no atestigua la presencia de Arendt entre los alumnos matriculados con Husserl en ningún curso de los años 20. Pero la gran biografía de Arendt firmada por su antigua alumna Elisabeth Young-Bruehl sí sostiene, con laconismo, que la joven estudiante se trasladó a Friburgo inmediatamente después de tener que abandonar Marburgo y que residió allí durante el semestre de invierno $1925-26 .{ }^{2}$ Ursula Ludz, editora de la correspondencia Arendt-Heidegger, señala, sin embargo, que Arendt no llegó a Friburgo hasta el curso siguiente, es decir, hasta el semestre lectivo de invierno 1926-27, o sea, después de haber estudiado ya un año completo en Heidelberg con Jaspers y antes de retornar de nuevo a la ciudad del Néckar. La correspondencia publicada entre Arendt y Heidegger no contradice esta posibilidad, pues en ella se acredita sólo la intención de Heidegger en mayo de 1925 de recomendarla como alumna ante Husserl y la de Arendt de aceptar tal recomendación; sin confirmación de que tal propósito llegase a cumplirse en ese momento. ${ }^{3}$ Y la más reciente biografía de Laure Adler, Dans les pas de Hannah Arendt, sostiene por su parte que Arendt no deja la ciudad del Lahn hasta la primavera de 1926, con lo que ella podría haber asistido en la capital de la Selva Negra al semestre de verano de ese mismo año; ${ }^{4}$ pese a contar con nuevas fuentes documentales y orales, Adler no deja constancia de cuál es el fundamento de su apreciación. Cada una de las tres autoras propone, en suma, un momento temporal y lectivo distinto para el paso de Arendt por Friburgo. $\mathrm{Y}$, en fin, por si en este pequeño lío faltara alguna posibilidad, Elisabeth Ettinger desplaza a Arendt directamente de Marburgo a Heidelberg sin pasar ni antes ni después por Friburgo. ${ }^{5}$ Claro que esta última opción sí tropieza con el contenido de la entrevista personal que Arendt concedió a la Televisión de Alemana Occidental en 1964. Allí se le formuló la siguiente pregunta: "Usted estudió en Marburgo, Heidelberg y Friburgo, teniendo por profesores a Heidegger, Bultmann y Jaspers. Filosofía como materia principal, y teología y griego como materias secundarias". 6 Y la entrevistada no corrigió entonces la formulación de la pregunta, pese a que ninguno de los tres pensadores citados profesaba en Friburgo durante el período de sus estudios universitarios. Esta no corrección podría así tener cierto valor indiciario del paso efectivo por Friburgo, aunque no despeje la duda acerca de la fecha precisa.

2 Hannah Arendt, Valencia, Alfons el Magnànim, 1993, pp. 89, 98.

3 Ursula Ludz (ed): Hannah Arendt/Martin Heidegger, Correspondencia 1925-1975, Barcelona, Herder, 2000, p. 33. La opinión de Ludz sobre la estancia de Arendt en Friburgo puede leerse en esta misma obra, p. 261.

4 Dans le pas de Hannah Arendt, París, Gallimard, 2005, pp. 64-67.

5 Hannah Arendt y Martin Heidegger, Barcelona, Tusquets, 1996, p. 44.

6 Vid. Ensayos de comprensión 1930-1954 -trad. de Agustín Serrano de Haro-, Madrid, Caparrós, 2005, p. 25. 
La incertidumbre sobre este aspecto biográfico no pasa de ser un mero detalle. Pues lo que indudablemente merecería un trabajo riguroso de análisis y discusión es la confrontación teórica entre la fenomenología de Husserl y la obra teórica de Arendt. Al cabo, la propia Arendt se entendía a sí misma como "una especie de fenomenóloga", así fuese para añadir al punto "pero. cuidado! no al modo de Hegel o de Husserl".: En qué consiste entonces, en sus rasgos generales, la fenomenología "al modo de Arendt"? Se trata del primado de la experiencia vivida en todo ejercicio de pensamiento teórico, o se trata de la adopción de un enfoque eidético a la hora de distinguir los órdenes de la vida activa y de deslindar las formas de los fenómenos políticos?.Podría incluso llegar a tratarse de la utilización de categorías básicas del análisis de la intencionalidad - sentido y horizonte de sentido, mundo de la vida, constitución intersubjetiva de la realidad -? La alergia de Arendt a las discusiones metodológicas hace que apenas contemos con precisiones a este respecto. Pero a falta de referencias explícitas, cabría quizá señalar dos grandes ámbitos de problemas en que, más que una coincidencia o convergencia de pareceres entre ambos interlocutores, ocurre que la obra admirable de Arendt entraña un poderoso desafío para la de Husserl y viceversa.

El primer ámbito residiría, a mi entender, en la peculiar fenomenología de la vida activa que Arendt desarrolla con extraordinario aliento descriptivo y ambición categorial. En este ámbito, la pregunta orientadora se parecería a la siguiente: la gran tematización arendtiana de la vida activa en labor, trabajo y acción, puede en verdad sostenerse sin una analítica suficiente de la vida intencional y, por tanto, sin una dilucidación original del aparecer del mundo? La articulación tripartita de la acción, se deja comprender fuera de la dinámica intencional y de su peculiarísima correlación? Yo no albergo dudas de que los análisis arendtianos de la labor y del trabajo encierran contribuciones verdaderamente relevantes para el esclarecimiento de la experiencia prepredicativa e incluso preperceptiva, que quedan decididamente situadas en un horizonte pragmático. Mas justamente por ello resulta imprescindible el contraste del enfoque de Arendt con la exploración husserliana de la receptividad intuitiva y de la pasividad sensible, es decir, de la experiencia del cuerpo propio y de la conciencia interna del tiempo

En relación con estos problemas, tendría también sịngular importancia el empeño de Arendt por analizar la acción humana fuera y lejos del dominio de la voluntad, o sea, bajo una exclusión consciente, a la vez metódica y ontológica, de los fenómenos de la voluntad. De acuerdo con ella, ni la labor del cuerpo ni el trabajo de las manos ni la praxis intersubjetiva responden en rigor a una tensión volitiva, ni descansarían sobre dimensiones propiamente volitivas de la intencionalidad. Se trata aquí de la muy arriesgada apuesta de Arendt en punto a que la voluntad es en general un concepto derivativo y sobreaña- 
dido a la experiencia del mundo; la voluntad y la libertad de la voluntad serían conceptos históricamente tardíos, inseparables de sus raíces paulinas - como intenta mostrar La vida del espíritu - y cuya problematicidad condena al pensamiento moderno a naufragar en la cuestión del libre albedrío. ${ }^{8}$ Pero esta enorme afirmación merece acreditarse no sólo en las fuentes hermenéuticas de las cartas de Pablo, sino en las mismas fuentes fenomenológicas de que Arendt dice beber.

El otro gran ámbito de encuentro y confrontación entre ambas figuras admirables discurre, sin embargo, en la dirección contraria. Ahora es una aportación característica de Arendt la que pone en cuestión enfoques básicos de la obra madura de Husserl. Estoy pensando, desde luego, en el descubrimiento y tematización del totalitarismo, o, como se ha podido decir, en la sorprendente "fenomenología del totalitarismo" que Arendt llevó a cabo para incomprensión general de su época. La interpelación podría formularse así: en la visión husserliana de la cultura europea y de la Historia del saber, de rasgos tan acentuadamente teleológicos, existe algún modo de pensar la novedad inaudita de los movimientos y regímenes totalitarios? La pretensión ideológica de los totalitarismos perseguía refundar las condiciones estructurales del mundo de la vida y del mundo de la Historia, al punto de que los seres humanos individuales resultaran superfluos y la condición humana, capaz de pensar y actuar con sentido, fuera el factor a eliminar en la construcción ideológica de un universo alternativo. Y este designio, al menos en su versión más radical: la del nazismo, se frustró sólo de manera contingente, por circunstancias y avatares bélicos, y sin que ninguna institución ni tradición europeas demostrasen ni la clarividencia ni la resolución necesarias para resistirlo. El mal que dimana del universo de los campos de concentración es a la vez imperdonable e incastigable, como si escapara a toda posible respuesta intencional, personal o intersubjetiva, que quisiera dar cuenta de él. No es por tanto este mal radical una contraposibilidad práctica, un imposible que la Historia ha hecho real y del que ninguna comprensión de la intersubjetividad puede hacerse cargo? La contraposibilidad se ha realizado en el tiempo histórico, aunque no llegara a consumar el cierre definitivo de la Historia, mas su dominio no fue vivido en buena medida como catástrofe ni como novedad inaudita, sino desde ese otro fenómeno desconcertante de la "banalidad del mal"; entre los agentes de la destrucción y entre amplias capas de la población se miró, se sintió y aceptó como normal lo que quebraba todo mundo normal de la vida.

En suma, la lucidez extrema de la descripción arendtiana del totalitarismo parece poner en evidencia lo deficitaria que resulta la fenomenología trascendental a la hora de comprender el alcance real y potencial del mal y a la hora de advertir la ceguera individual y colectiva de los seres humanos ante él. Y si

8 He considerado esta cuestión en "La epístola a los Romanos según Arendt", en: Nuevas teologías políticas. Pablo de Tarso en la construcción de Occidente, Reyes Mate y J. A. Zamora (eds.). Barcelona, Antrophos, 2005, p. 95-104. 
fuera certero el diagnóstico de Arendt de que la derrota de los totalitarismos no disuelve los elementos totalitarios que habitan constitutivamente el mundo contemporáneo y horadan su cultura, ese mal radical, que es un "todo de mal y mal del todo" (en la expresión de Emil Fackenheim), no deja de rondar a nuestro mundo y de constituir en él la posibilidad incierta que anula toda posibilidad, toda teleología, todo mundo de la vida.

Ninguno de estos dos enormes y decisivos ámbitos de discusión e interpelación será objeto de mi examen en las páginas que siguen. Me propongo una tarea preliminar mucho más sencilla, cual es la de señalar la idea general que la propia Arendt se hizo del pensamiento husserliano: cómo se representó Arendt la fenomenología de Husserl para al menos sostener que la suya propia respondía a otro estilo y a otros principios? Husserl no aparece citado una sola vez, es verdad, en La condición humana. "Igual que Heidegger", podría quizá argüirse si no fuera porque en el caso del autor de Ser y tiempo fueron razones y motivos personales los que desaconsejaron reconocer la deuda intelectual. Y en La vida del espíritu Husserl apenas merece alguna mención ocasional, a diferencia de Heidegger, que, aquí ya sí, es mencionado casi por todos lados. Existe, sin embargo, una señalada e importante excepción a esta ausencia de Husserl en la obra arendtiana. Pues el ensayo de 1946 "Qué es la filosofía de la existencia?", que constituye el primer escrito filosófico de Arendt desde la lejana tesis doctoral, y a la vez el texto más duro hacia Heidegger que salió nunca de su pluma, sí dedica un apartado íntegro al pensamiento de Husserl. ${ }^{9}$ En él se examina la novedad de la fenomenología, su contexto intelectual inmediato, su alcance y límites. Y, a mi juicio, ésta que es la única toma explícita de postura de Arendt respecto al fundador de la fenomenología resulta verdaderamente original y aguda.

2. Arendt caracteriza sorprendentemente a la fenomenología como un nuevo clasicismo y a su fundador como un clasicista. Ofreciendo la siguiente definición de clasicismo: "el intento llevado hasta el final de que una imitación rigurosa de lo clásico - y esto significa: del tener el hombre su hogar en el mundo - conjure una nueva patria para el hombre a partir de este mundo que se ha vuelto inhóspito". ${ }^{10}$ En el contexto del ensayo, lo inhóspito del mundo se remonta a la demolición kantiana del viejo concepto de ser. La antigua coincidencia entre esencia y existencia, en virtud de la cual lo que el hombre piensa con necesidad existe por fuerza, y lo que existe es por tanto comprensible y es de suyo racional, esta coincidencia preestablecida entre la esencia pensada o pensable y la existencia dada o dable ha cedido, ha caído; los hallazgos inauditos de la estructura antinómica de la razón humana y de la construcción trascendental de la experiencia la hacen imposible. Arendt insiste en que la acogida del hombre en el ser ha quebrado con la crítica kantiana: no hay ya una

9 Ahora puede leerse en Ensayos de comprensión 1930-1954, p. 203-231.

10 Op. cit., p. 205. 
pertenencia segura del hombre a una realidad dotada de sentido. No se trata sólo de una nueva etapa crucial en la desdivinización del mundo, sino también de un punto sin retorno respecto de la idea antigua de cosmos y respecto de toda idea sustantiva de verdad; todo lo que no es realidad individual ha quedado en cuestión en punto a su sentido y existencia, y el hombre como existencia consciente se sabe ahora extraño, excéntrico a toda legalidad general que pueda abarcarle y sostenerle. Con todo, Arendt apunta al hecho de que Kant dejó el trabajo a medias, pues él no destruyó la noción del ser como lo que está dado o predado, ni con ella la noción tradicional de la filosofía como consideración teórica, como teoría; de aquí que Kant aún pudiera aceptar, es decir, aún pudiera conformarse con que la libertad del hombre existe como mero factum en el seno de una totalidad que le es ajena e indiferente. En Schelling, que sería el primer filósofo contemporáneo, o sea, el primer pensador que consuma la escisión del existir respecto de toda comprensión teórica de él, rige ya, en cambio, la urgencia a "habérselas con el verdadero señor del Ser", la inquietud global de no poder conformarse con lo dado; pues Schelling sí siente que el hombre, al topar con su existencia irreductible y su libertad sin ley, ha perdido, por lo mismo, toda su realidad y sólo puede recuperarla acogiéndose de nuevo a Dios

Pero vengamos entonces a la fenomenología husserliana y a su supuesto clasicismo. De acuerdo con Arendt, la fenomenología se apartaría de la línea filosófica Schelling-Kierkegaard-Heidegger, en la medida en que el descubrimiento de la intencionalidad encierra la promesa de un nuevo hogar para el hombre en el mundo. Sin ningún tipo de restauración dogmática, sin soslayar la crítica kantiana, ocurre que la realidad del acto de conciencia arrastra consigo la certeza de su objeto intencional, y éste se impone como objeto real de mi conciencia, sea cual fuere la legalidad última del mundo. Tal sería para Arendt el sentido válido de la epojé, que salva la objetividad sin necesidad de comprometerse con ninguna tesis acerca de la realidad y de su origen. La corriente de conciencia vincula de manera infrangible el objeto y el acto, la cosa del mundo y la vida del hombre, bajo la única condición de justamente respetar su mutua irreductibilidad y su mutua correspendencia. Para Arendt cobra además especial relevancia el hecho de que Husserl proceda en su análisis de la conciencia a partir de correlatos individuales de actos, de cosas desligadas de sus conexiones funcionales y significativas. Ella llega incluso a afirmar que el programa fenomenológico de mathesis universalis pasaba por recomponer el mundo hecho pedazos a partir de estos términos intencionales discretos, aislados, inconexos.

A esta luz, Arendt se atreve a establecer una curiosa y estrecha afinidad entre "el sentimiento vital" que mueve a la fenomenología y que se plasma en el lema "A las cosas mismas", y un pensador, un escritor, que, aunque contemporáneo de Husserl, ha pasado desapercibido para la literatura fenomenológica. Se trata de Hugo von Hofmannstahl, y en particular de la famosa carta 
de Lord Chandos en que anuncia a Francis Bacon su renuncia a la escritura. La bellísima declaración de Hoffmanstahl en contra de las grandes palabras ${ }^{11}$ y a favor de las cosas pequeñas como las únicas que guardan el misterio de la realidad y despiertan en nosotros la antigua ternura por el mundo, está salpicada, ciertamente, de resonancias fenomenológicas. Los humildes objetos cotidianos - el rastrillo abandonado, el cementerio pobre, el lisiado en medio de un camino - se alzan casi de repente a "vaso de la revelación"; ellas cobran tal plenitud de presencia, tal abundancia de sentido cumplido, que las palabras - las intenciones signitivas vacías, podría aquí recordarse - se retiran, y dan paso a una "ingente participación" que es "mucho más y mucho menos que una compasión" ilimitada hacia todo lo que hay. Las cosas mismas de la fenomenología recuperadas justo en virtud de su acto de aparecer son - dice Arendt - estas mismas cosas pequeñas de Hofmannstahl de las que el poeta puede decir "todo estaba en mí", por más que este enigmático embelesamiento con ellas no remita ni a mi espíritu ni a mi cuerpo. En atención a esta plenitud de presente y de presencia se abriría entonces una posibilidad de rehumanizar un mundo inhóspito, es decir, la posibilidad de alentar un nuevo clasicismo.

Pero el análisis de Arendt da entonces un último paso, que mostraría por qué este camino filosófico era inviable, era sólo un conjuro "en un tiempo cuyo único patrimonio es que en él todo conjuro fracasa". ${ }^{12} \mathrm{Y}$ es que bajo el nuevo clasicismo se ocultaría una antigua hybris. La serena objetividad del fenómeno, del objeto pero intencional, serviría en realidad, en el pensamiento de Husserl, para tranquilizarnos a propósito de la fuente misma de intranquilidad e inhospitalidad del mundo contemporáneo. Pues la condena que pesa sobre el hombre, la de tener que "decir sí a un ser que él no ha creado y que le es ajeno", la subsana Husserl - añade Arendt - transformando el ser-ajeno de lo real en ser-consciente, y alimentando así la pretensión imposible de que el hombre, superada toda exterioridad, llegue a ser creador de sí y creador del mundo. Dicho de otro modo, Arendt entiende el concepto de constitución intencional como una segunda creación, y por tanto como una hybris que frustra la vuelta al clasicismo y revela la imposibilidad para el hombre del siglo XX de reconciliarse con la realidad: lo que está dado es sólo lo que el hombre secretamente ha constituido.

El posible lector de Arendt que sea también antiguo lector de Husserl, y cuya representación en este momento he de ejercer yo mismo, puede preguntarse, con todo, si la interpretación arendtiana del clasicismo fenomenológico y de su fracaso se sostendría en sus mismos términos si la hija de Heidegger o hija de Tracia tuviese que revisar algún aspecto capital de su exposición. Pues, por una parte, no son ciertamente objetos aislados, cosas inconexas o de conexiones muy circunscritas, los que, de acuerdo con la meditación husserliana,

11 Arendt dice de las "grandes palabras", pero "Una carta" se refiere a las palabras en general.

12 Op. cit., p. 205-206. 
aparecen a la corriente objetivadora de mi conciencia. La modestia infinita del aparecer, la verdad del fenómeno como dato original, va de la mano de que la manifestación radical y fundante es la del mundo ancho y ajeno. El mundo es como tal el aparecer primero, fundamental; "el vaso de la revelación" rebosa mundo, y yo mismo no puedo difuminar esta plenitud de presencia, que justamente define mi vida y la emplaza, la sitúa. Pero, en segundo lugar, la presencia indudable del objeto, de la cosa, del mundo, no obsta para que la mirada fenomenológica repare al mismo tiempo en el aparecer en cuanto tal, en el modo irreductible de darse la cosa. Un modo intuitivo (todo un sistema de modos) que no es construcción arbitraria del yo sino exigencia vinculada a la índole propia del objeto; pero que no por ello es tampoco una propiedad objetivable de la cosa ni la pura traslación de la cosa a un ámbito neutro de presencia. La perspectiva como tal forma parte constitutiva del aparecer, y no hay objeto posible cuya donación esté libre de un sistema de perspectivas, de tal forma que entre la cosa que aparece indudable y el aparecer efectivo de la cosa se establece la tensión sustantiva y fecunda que el análisis fenomenológico trata por doquier de describir. Es difícil valorar si estos dos matices fundamentales -lo que aparece ante todo es el mundo; el aparecer responde a una legalidad que no es puramente subjetiva ni puramente objetiva- permiten escapar al dilema entre el clasicismo de un mundo ajeno y la desmesura de un yo creador o cocreador. Entre el ser y el pensar hay, en todo caso, por así decir, una tercera cosa: el aparece. 